\title{
Garantía de derechos humanos de personas con discapacidad en el cantón Azogues provincia del Cañar de Ecuador
}

\section{Human Rights' Guarantee of Disabled People in the Canton Azogues, Cañar Province, Ecuador.}

\author{
Luis Bolívar Marín Carangui ${ }^{1 *}$ y María Augusta Torres Rodas $^{1}$ \\ ${ }^{1}$ Universidad Católica de Cuenca, Ecuador \\ *lbmarinc@ucacue.edu.ec
}

DOI: https://doi.org/10.26871/killkana_social.v4i1.618

\begin{abstract}
Resumen
Los derechos humanos de las personas con discapacidad, se garantizan expresamente en la Constitución Política del Ecuador, Convenios y Tratados Internacionales y demás legislación interna; a pesar de lo antes expuesto, no se cumple con la protección integral a este grupo de atención prioritaria por parte del Estado, sociedad y familia. Esta situación obedece a la falta o incumplimiento de políticas públicas, con lo que se vulneran los derechos fundamentales en la protección, defensa y exigibilidad, y se afecta la calidad de vida, en especial de personas que tienen algún tipo de discapacidad. La investigación se realizó en coordinación con el Consejo Cantonal de Protección de Derechos del Cantón Azogues, Fundación para el Desarrollo Humano y la Universidad Católica de Cuenca, Sede Azogues. Se parte de la hipótesis que con el conocimiento del ejercicio de los derechos humanos y sociales de las personas con discapacidad y su realidad local, se pueden construir de manera participativa políticas públicas que respondan a la vulneración de derechos con la implementación de planes, programas y proyectos por parte de diferentes instituciones debidamente articuladas, planteándose el objetivo de: "Determinar el cumplimiento de la garantía de los derechos humanos de las personas con discapacidad del Cantón Azogues de la Provincia del Cañar”. Para el presente estudio se indagó en fuentes secundarias o bibliográficas y técnicas de recolección de datos como la observación, entrevistas y encuestas, con enfoque cualitativo y cuantitativo, en las que se evidencia que existe vulneración de derechos de los grupos de atención prioritaria en las diferentes parroquias del Cantón, y esto obedece a factores sociales, políticos y económicos. No existen mecanismos de control y seguimiento en el cumplimiento de políticas públicas, limitando el ejercicio y cumplimiento de los derechos fundamentales de este grupo de atención prioritario.
\end{abstract}

Palabras clave: discriminación de las personas con discapacidad; exclusión social; derechos humanos; culturas inclusivas.

\begin{abstract}
The human rights of disabled people are explicitly guaranteed in the Constitution, international agreements, treaties and other internal legislation; despite this, comprehensive protection for this priority group is not fulfilled by the State, society and family. This situation is the result of the lack of or failure to implement public policies, thus violating fundamental rights in terms of protection, defence and rigorousness, and affecting the quality of life, especially of the people who have some disability. The research was conducted in coordination with the Rights Protection Cantonal Council of the Azogues Canton, the Foundation for Human Development and the Catholic University of Cuenca, Azogues seat. It is based on the hypothesis that by knowing about the human and social rights of disabled people and their local reality; public policies can be created in a participatory manner to respond to rights' violation, by implementing plans, programs and projects executed by different institutions which are duly articulated. The objective is: "To determine compliance with the human rights guarantee of the disabled people in the Canton of Azogues in the Province of Cañar. For the present study, secondary or bibliographic and technical data collection sources such as observation, interviews and surveys were examined with a qualitative and quantitative approach. The results show that the priority groups' rights are being violated in the different parishes of canton, which is due to social, political, and economic issues. The lack of control and monitoring mechanisms in the implementation of public policies restricts the exercise and fulfilment of this priority group's fundamental rights.
\end{abstract}

Keywords: discrimination of disabled people; social exclusion; human rights; inclusive cultures. 


\section{Introducción}

Según (Asamblea Nacional del Ecuador, 2008) el artículo 35 enfatiza que, las personas con discapacidad son de prioritaria atención, lo que significa que el Estado Ecuatoriano a través de las diferentes instituciones públicas y privadas deben cumplir la ley y las políticas públicas a través de la asignación los recursos necesarios para la atención de éste grupo vulnerable, empero de aquello, en los diferentes niveles de gobierno, en su mayoría no ha sido efectivo; en este contexto, surge el cuestionamiento si la institucionalidad del Estado está efectivamente garantizando los derechos humanos de las personas con discapacidad en el marco de sus competencias, surgiendo las preguntas: ¿Se reconoce los derechos fundamentales de los grupos de atención prioritaria? ¿Se han eliminado las barreras culturales, simbólicas y arquitectónicas para el libre acceso social de las personas con discapacidad? ¿Se ha vulnerado derechos del buen trato de las personas con discapacidad? Interrogantes que han sido solventadas de acuerdo a los resultados de investigación.

Al respecto se tiene que, en Ecuador, concretamente en el Cantón Azogues de la provincia del Cañar, las personas con discapacidad no están siendo atendidas de forma integral como grupos de atención prioritaria, requiriendo contar con un diagnóstico participativo que permita establecer científicamente el grado de cumplimiento de estos derechos en el marco de la Constitución Política del Ecuador, Tratados y Convenios Internacionales, que permita direccionar políticas públicas de promoción, difusión, protección, defensa y exigibilidad de derechos a través de planes, programas y proyectos debidamente financiados, con el objetivo de garantizar la calidad de vida de este grupo de personas y sus familias.

El interés que tiene este aporte científico es que, en base a los hallazgos y problemática social, política y económica, promover la garantía de los derechos humanos de las personas de los grupos de atención prioritaria, especialmente de las personas con discapacidad a través de motivar procesos gestión institucional en la implementación, formulación y seguimiento de políticas públicas a nivel local en corresponsabilidad del Estado, sociedad y familias, mediante un trabajo sistémico y ordenado de redes interinstitucionales mediante la articulación y sinergias institucionales de recursos financieros, técnicos e inversión de los gobiernos autónomos descentralizados en cumplimiento del (Asamblea Nacional del Ecuador, 2011), invirtiendo presupuestos sociales como lo dispone el Art. 249 del COOTAD: "Los presupuesto para los grupos de atención prioritaria, no se aprobarán si en el mismo no se asigna, por lo menos, el diez por ciento (10\%) de sus ingresos no tributarios para el financiamiento de la planificación y ejecución de programas sociales para la atención a estos grupos prioritarios" (Asamblea Nacional del Ecuador, 2010), que garantice el derecho del buen vivir que generen impactos significativos y sostenidos en el Cantón Azogues y sus parroquias.
La ineficacia operativa en el cumplimiento de políticas públicas o la inexistencia de éstas en territorio, vulneran derechos de las personas con discapacidad, afectando la calidad de vida por la exclusión y desatención, constituyéndose una constante lucha histórica de estos grupos sociales y sus familias, consolidándose en algunas sociedades un modelo de derechos humanos, basados en la Convención Internacional de las Personas con Discapacidad, considerado como un verdadero avance en cuenta al desenlace histórico; empero de aquello, queda mucho por desarrollar y hacer cumplir.

La investigación se constituye como un proyecto de vinculación con la comunidad, con iniciativa del Gobierno Autónomo Descentralizado Municipal del cantón Azogues, Consejo Cantonal de Protección de Derechos, Fundación para el Desarrollo Humano, FENDEHU y la Universidad Católica de Cuenca Sede Azogues, en atención a una problemática de interés social, que permita proyectar propuestas de trabajo con impactos significativos en la calidad de vida de estos grupos de atención prioritaria en el Cantón. La investigación está sostenida por estudios y postulados de varios doctrinarios basado en principios universales que fundamentan que todas las con discapacidad tienen los mismos derechos en su dignidad, libertad e igualdad, con las mismas oportunidades; no deberían ser excluidos, ni discriminados, deben ejercer sus derechos tal y cual como lo hacen las personas sin discapacidad, tomando el concepto de la discapacidad entendida en su contexto social según (Saleh, 2004). Es mucho más que una mera condición, es una experiencia de opresión y de exclusión, pero los responsables de esta experiencia no son las personas con discapacidad sino la indiferencia y la falta de comprensión de la sociedad. Las personas con discpacidad son un desafío para el resto de la sociedad.

El estudio de investigación prueba la hipótesis que: Conociendo la situación actual de los derechos de las personas con discapacidad se promueven políticas públicas participativas con actores sociales estratégicos en la inclusión social, institucional, política y económica a través de la planificación e inversión en planes, programas y proyectos para grupos de atención prioritaria; para tal efecto, se cumple los objetivos de Investigar la situación actual en el ejercicio de los derechos humanos de las personas con discapacidad que residen en el cantón Azogues y sus parroquias rurales a fin de contar con un diagnóstico situacional de este grupo de atención prioritaria. Aportar conocimiento científico que generen condiciones para la elaboración de planes, programas y proyectos en atención a las personas con discapacidad y sus familias.

\section{Material y Métodos}

La investigación se desarrolló en las diferentes parroquias del cantón Azogues de la provincia del Cañar. Como fuente del estudio, se emplea una metodología de investigación de campo en los Gobiernos Autónomos Descentralizados Parroquiales-GAD's (grupos focales de diferentes co- 
munidades), viviendas, representantes de las personas con discapacidad intelectual, centros de formación ocupacional de personas con discapacidad intelectual.

La población referente para la investigación de campo se ha tomado como fuente la proporcionada por el Consejo Nacional para la Igualdad de Discapacidades del Ecuador - CONADIS, Ministerio de Salud Pública, del 2 de septiembre de 2018, en donde se establece que en el cantón Azogues existen 3036 con personas con discapacidad.

El método utiliza técnicas de recolección de datos como la observación y aplicación de herramientas de entrevistas y 237 encuestas de fácil aplicación que llevó a la verificación de la hipótesis del tema planteado con respecto al cumplimiento de los derechos humanos de este grupo de atención prioritaria.

Previo a la aplicación de encuestas y entrevistas en diferentes parroquias, se realizó una fase de investigación previa a través de visitas a los Gobiernos Autónomos Descentralizados Parroquiales, Ministerio de Inclusión Económica y Social, Centros de Salud, Gobierno Autónomo Descentralizado Municipal de Azogues, Consejo Nacional de Discapacidades, entre otras entidades,

Como investigación de fuentes secundarias o bibliográficas, se toma en consideración a Hernández Sampieri, autor del libro Metodología de la Investigación Científica, sexta edición, en la que define a la Investigación como "un conjunto de procesos sistemáticos y empíricos que se aplican al estudio de un fenómeno o problema social" (Hernández Sampiere, 2014)

Para (Hernández Sampiere, 2014) durante el siglo XX surgieron dos aproximaciones a la investigación: el enfoque cuantitativo y el enfoque cualitativo. En términos generales, los dos enfoques emplean procesos cuidadosos, sistemáticos y empíricos para generar conocimiento. La definición de investigación es válida tanto para el enfoque cuantitativo como para el cualitativo. Ambos constituyen un proceso general que a su vez integra diversos procesos.

La investigación bibliográfica parte del análisis del Plan Nacional de Buen Vivir o Sumak Kawsay que se encuentra establecida en la Constitución de la República del Ecuador en especial atención a las personas que por su condición se encuentren en situación de vulnerabilidad, con el fin de que las mismas conozcan y hagan efectivos sus derechos. La recolección de información se realizó mediante la exploración de libros de autores nacionales e internacionales, tomando en consideración un marco históricológico, que permitió comprender el origen del Derecho a la inclusión y las luchas sociales entorno a los tiempos pasados, su evolución y su reconocimiento como tal en el Ecuador. Se utilizó el método explicativo, que dio a conocer la realidad que viven las personas con discapacidades en relación a lo que indica la normativa, la Ley Orgánica de Discapacidades sobre este tema. -El método exegético, permitió el estudio y análisis de varias de las normas, en los cuales tenemos plenamente amparados los derechos de las personas con discapacidades.
En las técnicas de campo, la aplicación de la encuesta se basa en la Población-Muestra, con preguntas cerradas que facilitaron la tabulación de los datos para para establecer conclusiones más precisas de investigación.

\section{Resultados}

De los resultados generales de la investigación se puede afirmar que, a pesar que el Ecuador es un Estado garantista de derechos y justicia, social no se ha cumplido de forma integral los derechos humanos de las personas con discapacidad como grupo de atención prioritaria, siendo en muchos casos excluidos e invisibilizados por políticas ineficientes y falta de voluntad o cultura política social en la garantía de derechos de los grupos de atención prioritaria.

En el cantón Azogues provincia del Cañar, de acuerdo al Consejo Nacional para la Igualdad de DiscapacidadesCONADIS- Ministerio de Salud Pública, a fecha de 02 septiembre de 2018, existen 3036 con personas con discapacidad, de los cuales el $53.39 \%$ son hombres y $46,60 \%$ son mujeres. El $3.46 \%$ están comprendidos entre edades de 0 a 3 años; el $1.22 \%$ entre 4 a 6 años; el $4.74 \%$ entre 7 y 12 años; $6.65 \%$ entre 13 a 17 años; el $15.28 \%$ entre 18 a 20 años; 45,69\% entre 30 y 65 años; y mayores de 65 años el $25.96 \%$.

Del total de personas con discapacidad, el 38,24\% tienen discapacidad física; el $13.34 \%$ auditiva; $15.48 \%$ visual; $8.17 \%$ psíquica-social; $y$, el 24,77\% tiene discapacidad intelectual.

La planificación del estudio direccionaba a realizar encuestas y entrevistas a la mayor cantidad de instituciones públicas y privadas del cantón Azogues que presten servicios a personas con discapacidad, empero de aquello, no se pudo acceder a datos de información por la confidencialidad que justificaban los representantes legales de algunas de estas éstas; obligando a direccionar el trabajo de investigación a todas la juntas parroquiales del Cantón, como Rivera, Taday, Pindilig, Chuquipata, Guapán, San Miguel de Porotos, Cojitambo, Luis Cordero, Javier Loyola, que finalmente constituyó un valor agregado en razón que se pudo medir el grado de cumplimiento de los Derechos Humanos de las personas con discapacidad sobre todo la cobertura de servicios y conocimiento de los derechos en las zonas rurales. De esta manera se realizaron 237 encuestas a personas con discapacidad en las diferentes parroquias referidas, divididas entre 59 mujeres y 178 hombres.

De las 237 personas con discapacidad encuestados, el $29 \%$ son casados, $9 \%$ son divorciados, $47 \%$ solteros, $4 \%$ unión de hecho, $11 \%$ son viudos. Se puede observar que el $53 \%$ de este grupo de atención prioritaria tuvo la oportunidad el derecho de formar una familia, en tanto que el resto de encuestados en su mayoría señala que no han formado una familia por su condición.

Un dato importante que llama la atención es que, el $76 \%$ de personas con discapacidad encuestadas no estudian por dificultad de acceso a la educación. En algunos casos las familias se han visto en la obligación de retirar a 
sus hijos de las instituciones educativas porque los procesos de enseñanza-aprendizajes no son adaptables para las necesidades de algunas discapacidades. Además, existen familias que no apoyan el ingreso de sus hijos a centros educativos por el temor al fracaso, exclusión y vulneración de derechos por sus pares. Es de señalar que existen grados de discapacidad elevados, que requieren centros especializados para su atención que no existen en las parroquias o comunidades, únicamente en el centro cantonal que incluso son escasos y de difícil acceso por la distancia y limitación de recursos económicos; es así que, considerando un rango de edad propio para estudiar, 44 personas con discapacidad comprendidas entre 6 y 26 años de edad, apenas el $61 \%$ estudia.

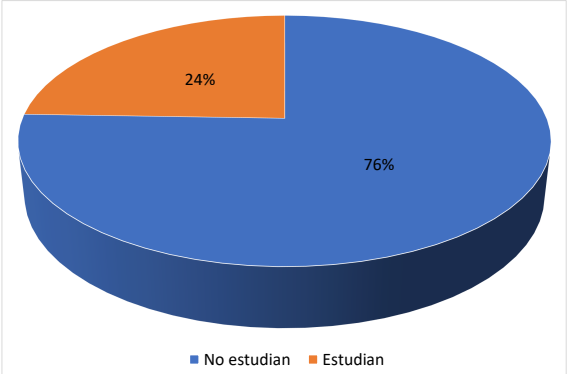

Figura 1: Personas con discapacidad que estudian Fuente: Investigadores

De las personas encuestadas, el $41 \%$ de personas con discapacidad tienen instrucción primaria; el $8 \%$ instrucción secundaria; el $14 \%$ educación superior; y, el $37 \%$ no tienen ninguna educación, a este último grupo se podría considerar en condiciones de doble vulnerabilidad, que, por sus condiciones son excluidos con mucha frecuencia por parte del Estado, sociedad y la familia.

Con respecto a tipo de discapacidades, el $29,1 \%$ es auditiva; el $21,5 \%$ es física; el $8,0 \%$ tiene problemas de lenguaje; el 5,1\% tiene más de una discapacidad; y, el $18,1 \%$ tienen discapacidad visual. De las diferentes discapacidades, el 22,8\% tienen entre $20 \%-39 \%$ de discapacidad; el $24,1 \%$ entre $40 \%-49 \%$; el 7,6\% tiene entre $50 \%$ $59 \%$; el $21,5 \%$ entre $60 \%-74 \%$; el $18,1 \%$ entre $75 \%$ $84 \%$; y, el 5,9\% tiene entre $85 \%-100 \%$ de discapacidad.

El $66 \%$ de personas con discapacidad no tiene trabajo, pudiéndose establecer que muchos de éstos no pueden acceder a un trabajo a pesar de tener capacidades o grados aceptables de discapacidad para tener una relación laboral, sin embargo son excluidos a pesar que la ley laboral exige la contratación de personas con discapacidad de acuerdo al el artículo 42 numeral 33 del Código del Trabajo que establece que tanto las instituciones públicas como privadas que tenga de 25 empleados, determina que el empleador público o privado, que cuente con un número mínimo de veinticinco trabajadores, tiene que contratar una persona con discapacidad, considerando condiciones físicas, intelectuales y aptitudes personales en el marco de los principios de equidad, diversidad en la discapacidad. Desde el año 2009, constituye como imperativo legal la contratación del $4 \%$ de personas con discapacidad de la totalidad de trabajadores. (Ministero del Trabajo de Ecuador, 2005). (Ecuador, Ley Orgánica de Servicio Público, 2010), Art. 64, todas las instituciones que tengan más de veinte y cinco servidores, de igual forma, tienen obligación de tener relación laboral con personas con discapacidad, exigiendo acciones afirmativas como demanda la Constitución del 2008, que se contrate obligatoriamente hasta el $4 \%$ del total de servidores. Al respecto se puede afirmar que el Ministerio de Trabajo, no tiene, o teniendo procesos sistemáticos de seguimiento en la aplicación de la ley y políticas públicas, no lo hacen eficientemente, afectando directamente oportunidades que por derecho le corresponde.

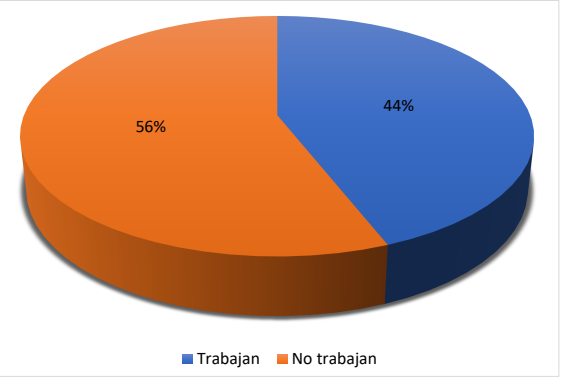

Figura 2: Personas con discapacidad que laboran Fuente: Investigadores

De las personas encuestadas, el $26 \%$ de las personas no conocen los derechos de las personas con discapacidad; el $31 \%$ conoce un poco; y el $43 \%$ si conocen. Es decir, el $57 \%$ de personas con discapacidad no conocen los derechos que les asiste la Constitución de la República del Ecuador y la Ley Orgánica de Discapacidades. Con respecto a los derechos que les corresponde de acuerdo a su discapacidad, el $72 \%$ no conocen los derechos que les asiste. Claramente se puede afirmar que no existen políticas de promoción y difusión de los derechos, generando desconocimiento en la aplicación y ejercicio de derechos.

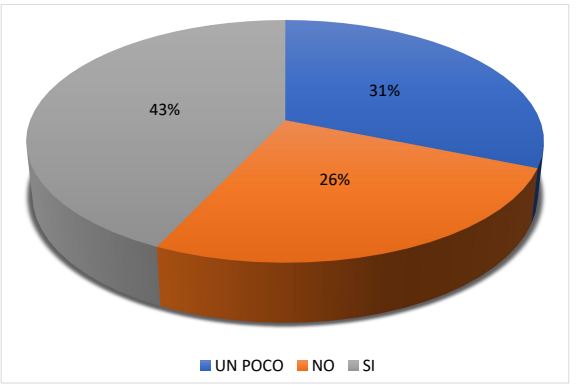

Figura 3: Conocimiento sobre los derechos de las personas con discapacidad

Fuente: Investigadores

Con respecto a la discriminación a la que han sido, se puede observar que el $11 \%$ ha sido discriminado por varias personas como amigos, compañeros inclusive por su 
propia familia; el $7 \%$ por personas de la comunidad en la que convive; el $4 \%$ por su propia familia; el $3 \%$ por parte de sus amigos; el $6 \%$ por sus compañeros; en tanto que el $69 \%$ no ha sentido ninguna discriminación por parte de otra persona. Resultados que recalcan la necesidad de sensibilización y capacitación en valores en el marco de derechos.

En relación a los beneficios de los servicios por parte del Estado, el $34,78 \%$ de las personas que padecen discapacidad no reciben ningún beneficio. El $36 \%$ recibe bonos, $9 \%$ recibe ayuda de la fundación Joaquín Gallegos Lara; y, el $6 \%$ les apoya la fundación Manuel Espejo, demostrando que la cobertura de los servicios públicos no atiende a los grupos vulnerables más distantes, quedando en total exclusión, afectando todos los derechos de dignidad humana, incumpliendo principios de justicia e igualdad.

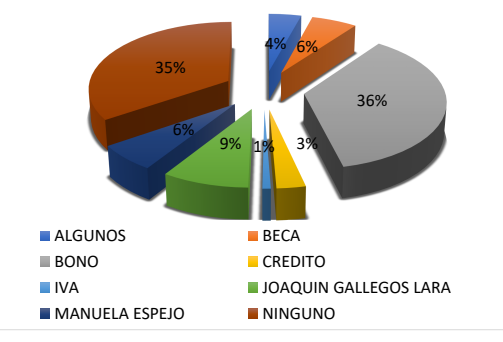

Figura 4: Beneficios recibidos por parte del estado

Fuente: Investigadores

En el tema de seguridad social, el $52 \%$ de las personas con discapacidad cuentan con el seguro social; y, el $48 \%$ no cuentan con este servicio. De las personas que tienen seguridad social el $14 \%$ cuentan con el seguro voluntario; el $14 \%$ de las personas cuentan con el seguro con relación laboral; el $14 \%$ cuentan con el seguro campesino; el $8 \%$ de las personas cuentan con el seguro privado; y el $2 \%$ tienen más de uno de los seguros. Existe un grupo considerable que no cuenta con seguridad social que puede afectar el derecho universal de la salud.

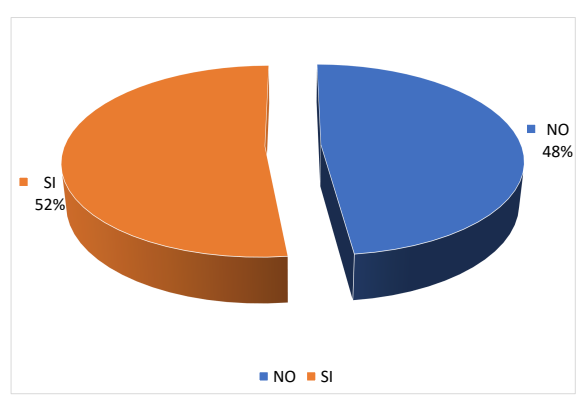

Figura 5: Beneficio de seguro social

Fuente: Investigadores

La prestación de servicios públicos y privados en relación al cumplimiento de los derechos, el $30 \%$ de las personas con discapacidad manifiestan que no reciben trato preferencial por la condición de su discapacidad en las instituciones del Estado, instituciones privadas, transporte, centros educativos, bancos, etc. Se confirma que el seguimiento a las políticas públicas, leyes de protección y defensa de los derechos para estos grupos de atención prioritario son ineficaces o simplemente las instituciones y autoridades no cumplen las responsabilidades de acuerdo a sus competencias y obligaciones.

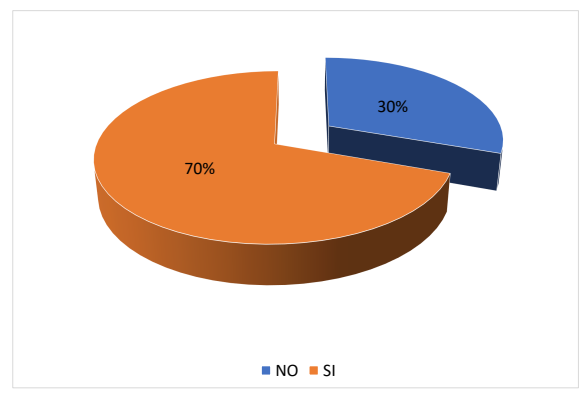

Figura 6: En la prestación de servicios públicos-privados y el cumplimiento del derecho de atención prioritaria a las personas con discapacidad

Fuente: Investigadores

El $71 \%$ de las personas con discapacidad encuestadas necesitan ayuda para desenvolverse como amigos, familiares o vecinos, recibiendo apoyo de sus padres $(21 \%)$, parientes $(36 \%)$, y de otras personas (43\%). Estas personas que brindan apoyo requieren conocer los derechos que le asisten a las personas con discapacidad para que sean actores claves en la protección, defensa y exigibilidad, para tal efecto, las estrategias de capacitación deben ser orientadas a todas la familias, comunidades e instituciones, es decir, corresponsabilidad del Estado, sociedad y familia.

\section{Discusión}

Según (Organización de las Naciones Unidas, 2006), en el artículo 1 señala que las personas con discapacidad son personas que tienen deficiencia, físicas, mentales, intelectuales o sensoriales a lo largo del tiempo y que la Convención quiere que éstas personas disfruten de todos los derechos humanos y de todas las libertades.

(Saleh, 2004) recuerda que la primera frase de la Declaración Universal de los Derechos Humanos adoptado por la Asamblea General de las Naciones Unidas en 1948 era que: "todos los seres humanos nacen libres e iguales en dignidad y derechos" sin embargo en la actualidad aún existen millones de personas con discapacidad cuyos derechos humanos se encuentran seriamente limitados o totalmente negados (p. 9).

$\mathrm{Al}$ respecto se debe señalar que si bien el tema de los derechos humanos y sociales de las personas con discapacidad en el mundo, en América Latina y en Ecuador, ha ido evolucionando, algunos autores e instituciones que se han referido al respecto, es el caso de las Naciones Unidas, coinciden en que: "las personas con discapacidad tienen 
pleno derecho a la igualdad de oportunidades y a la plena inserción social, sin embargo se requiere de un cambio cultural y conseguir este cambio, requiere sistemas jurídicos inclusivos y responsable para sostener una sociedad que queremos" (Organización de las Naciones Unidas, 2006)

(Eroles y Fiambarti, 2008) al ampliar el análisis, refriéndose a las personas con discapacidad y la discriminación, señalan que, la Convención Interamericana, sancionada por la Organización de Estados Americanos (1999), definía a la discriminación de las personas con discapacidad como: "Toda distinción, exclusión o restricción basada en una discapacidad anterior, presente o pasada que tenga el efecto o propósito de impedir el reconocimiento, goce y ejercicio por parte de las personas con discapacidad de sus derechos humanos y libertades" (p. 20).

(Palacios, 2008) "El Modelo Social de Discapacidad", sostiene que la discapacidad es una cuestión de derechos humanos y que en la actualidad, ésta afirmación no es cuestionada, sin embargo, hacia tiempos no muy lejanos la mirada hacia la discapacidad partía desde una concepción caritativa que no llegaba a comprender la complejidad social de este fenómeno.

Según (Asamblea Nacional del Ecuador, 2008), en el artículo 35 enfatiza en que, las personas con discapacidad son de prioritaria atención, lo cual significa que el Estado Ecuatoriano a través de las diferentes instituciones públicas y privadas deben asignar los recursos necesarios para la atención de este grupo vulnerable, empero de aquello, en los niveles de Gobierno Descentralizados, en su mayoría no ha sido efectivo, es por ello que se torna necesario en la actualidad cuestionarnos si es que efectivamente se están respetando los derechos humanos y sociales de las personas con discapacidad por parte de las instituciones públicas.

Según los autores referidos, concluyen que las personas con discapacidad tienen los mismos derechos en su dignidad y libertad e igualdad, con las mismas oportunidades; no deberían ser excluidos, ni discriminados, deben ejercer sus derechos tal y cual como lo hacen las personas sin discapacidad. La discapacidad entendida en su contexto social según (Saleh, 2004) es mucho más que una mera condición, es una experiencia de opresión y de exclusión, pero los responsables de esta experiencia no son las personas con discapacidad sino la indiferencia y la falta de comprensión de la sociedad. Las personas con discapacidad son un desafío para el resto de la sociedad.

De tal manera que es un proceso de sensibilización y voluntad política tanto a nivel de Estado, sociedad y familia, al igual que autoridades y sobre todo los gobiernos autónomos descentralizados cumplan las disposiciones del (Asamblea Nacional del Ecuador, 2011) garantizando los derechos de las personas discapacidad invirtiendo presupuestos sociales como lo demanda el Art. 249 del COOTAD: "Los presupuesto para los grupos de atención prioritaria, no se aprobarán si en el mismo no se asigna, por lo menos, el diez por ciento $(10 \%)$ de sus ingresos no tributarios para el financiamiento de la planificación y ejecución de programas sociales para la atención a estos grupos prioritarios".

Esto implica que las políticas públicas nacionales y locales deben cumplirse estrictamente en la garantía de los derechos de las personas con discapacidad en el cantón Azogues y sus parroquias. Los Consejo Cantonal de Protección de Derechos, CCPD, en el marco de sus competencias tienen la facultad de dar seguimiento a políticas públicas con circunscripción cantonal; $y$, en casos necesarios, formular políticas públicas en atención a mejorar la calidad de vida de los grupos de atención prioritaria a través de elaboración de agendas de política pública con enfoque intercultural, generacional, género, etc., que, de los resultados obtenidos en la investigación no cumplen con estas competencias que afectan directamente a todos los grupos de atención prioritaria.

Un componente trascendental en la gestión de los CCPD es observar, vigilar y activar mecanismo para exigir el cumplimiento de los derechos por parte de todas las instituciones públicas y privadas a través de redes o coordinaciones interinstitucionales, además, todas las competencias que describe la ley de Igualdad de Igualdad Intergeneracional y la Ordenanza del Sistema de Protección Integral de Derechos del Cantón Azogues.

Con los resultados del presente estudio se puede evidenciar que existe vulneración de derechos de los grupos de atención prioritaria en las diferentes parroquias del cantón Azogues, obedece a factores de cobertura en servicios, información, capacitación, desarticulación institucional. Por otra parte, no existen mecanismos de control y seguimiento al cumplimiento de políticas públicas por parte de las diferentes instituciones en territorio. Finalmente, el desconocimiento sobre derechos de las comunidades, familias y personas con algún tipo de vulnerabilidad que le hace parte de un grupo de atención prioritaria, limita el ejercicio y la exigibilidad en el cumplimento de los derechos fundamentales.

En este contexto, se puede recomendar los siguientes ejes de intervención:

Político, estructurar modelos de seguimiento de las políticas públicas cantonales mediante rendición de cuentas de todas las instituciones públicas y privadas en relación a cumplimiento y coberturas que permitan priorizar la atención en los grupos de mayor vulnerabilidad;

Gestión, diseñar y desarrollar programas y proyectos de información, sensibilización y capacitación a comunidades, familias y grupos de atención prioritaria en temas de cumplimiento y ejercicio de derechos humanos;

Comunicación, delinear una estrategia comunicacional de sensibilización e información sobre el cumplimiento y ejercicio de los derechos humanos de los grupos de atención prioritaria (prensa, radio, televisión, productos comunicaciones, etc.);

Aprobar políticas locales de gestión debidamente financiados para garantizar el cumplimiento especializado en 
derechos de los grupos de atención prioritaria de acuerdo a la problemática local;

Profundizar estudios de cumplimiento de derechos por cada grupo de atención prioritaria para garantizar una intervención efectiva y progresiva en el cumplimiento de las políticas públicas;

Conformar redes interinstitucionales, defensorías comunitarias, consejos consultivos, veedurías, observatorios de intervención para responder de forma diligente problemáticas de territorio con mayores impactos sociales;

Uno de los componentes y ejes fundamentales para el desarrollo de cualquier estrategia, planificación e intervención es el fortalecimiento del conocimiento de las competencias de los miembros del Consejo Cantonal de Protección de Derechos, CCPD; y sobre todo, compromiso y voluntad política de autoridades e instituciones con legalidad y legitimidad ciudadana por medio de resultados progresivos en la calidad de vida de estos grupos de atención prioritaria.

\section{Referencias Bibliográficas}

Asamblea Nacional del Ecuador. (2008). Constitución de la República del Ecuador. Quito: MATT, Corporación Jurídica S.A.

Asamblea Nacional del Ecuador. (2010). Ley Orgánica de Servicio Público. Quito: LEXIS.

Asamblea Nacional del Ecuador. (2011). Código Orgánico de Organización Territorial, Autonomía y Descentralización. QUITO: Asamblea Nacional del Ecuador.

Eroles, C., y Fiambarti, H. (2008). Los Derechos de las Personas con Discapacidad (Análisis de las Conven- ciones Internacionales y de la Legislación Vigente que los Garantiza). Buenos Aires: EUDEBA.

Hernández Sampiere, R. (2014). Metodología de la Investigación Científica. En Metodología de la investigación científica (p. 18). Mexico: MCGRAW HILL.

Ministero del Trabajo de Ecuador. (2005). Código de Trabajo del Ecuador. Quito: Ediciones Legales.

Organización de las Naciones Unidas. (2006). Convención de los Derechos de las Personas con Discapacidad. New York: ONU.

Palacios, A. (2008). Modelo Social de Discapacidad: Origen, Caracterización y Plasmación en la Convención Internacional Sobre los Derechos de las Personas con Discapacidad. Madrid: CINCA.

Saleh, L. (2004). Inclusión Social, Discapacidad y Políticas Públcias. Santiago: Carolina Bañados.

Recibido: 15 de septiembre de 2019

Aceptado: 10 de febrero de 2020

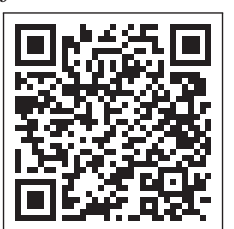


